\title{
Targeting epigenetic regulation of osteoclastogenesis to prevent bone loss
}

$\mathrm{N}$ ew research published in Nature Medicine sheds light on the pathophysiology of bone resorption, and suggests that DNA methylation could be a promising therapeutic target in the treatment of bone disorders such as osteoporosis.

In their investigation of epigenetic regulation in osteoclasts, the researchers first identified that expression of the DNA methyltransferase Dnmt3a is increased during osteoclastogenesis. Experiments in embryonic stem cells then determined that Dnmt3a has an important role in receptor activator of nuclear factor $\kappa \mathrm{B}$ ligand (RANKL)-induced osteoclast differentiation.

"To investigate the in vivo significance of Dnmt3a, we generated osteoclast-specific knockout mice by crossing Dnmt3a flox mice and osteoclast-specific Cre lines," explains Keizo Nishikawa, lead author of the study. "Dnmt3a knockout mice exhibited a high bone mass phenotype due to abnormality in osteoclasts." Compared with control littermates, mice with osteoclastspecific Dnmt3a deficiency had increased bone mass and normal parameters of bone formation, but low numbers of osteoclasts and decreased bone resorption.

To explore whether modulating the activity of Dnmt3a could be used to control osteoporosis, the researchers turned to animal models of bone-resorptive disorders. By using Dnmt3a knockout mice to create an ovariectomy (OVX)-induced model of postmenopausal osteoporosis, they were able to investigate the role of Dnmt3a in the pathological activation of osteoclastogenesis. "The bone mass in Dnmt3a knockout ovariectomized mice was higher than that in control ovariectomized mice," Nishikawa relates. "Furthermore, the increase in osteoclast number induced by ovariectomy was much lower in Dnmt3a knockout mice, suggesting a key role of Dnmt3a in the pathological activation of osteoclastogenesis."
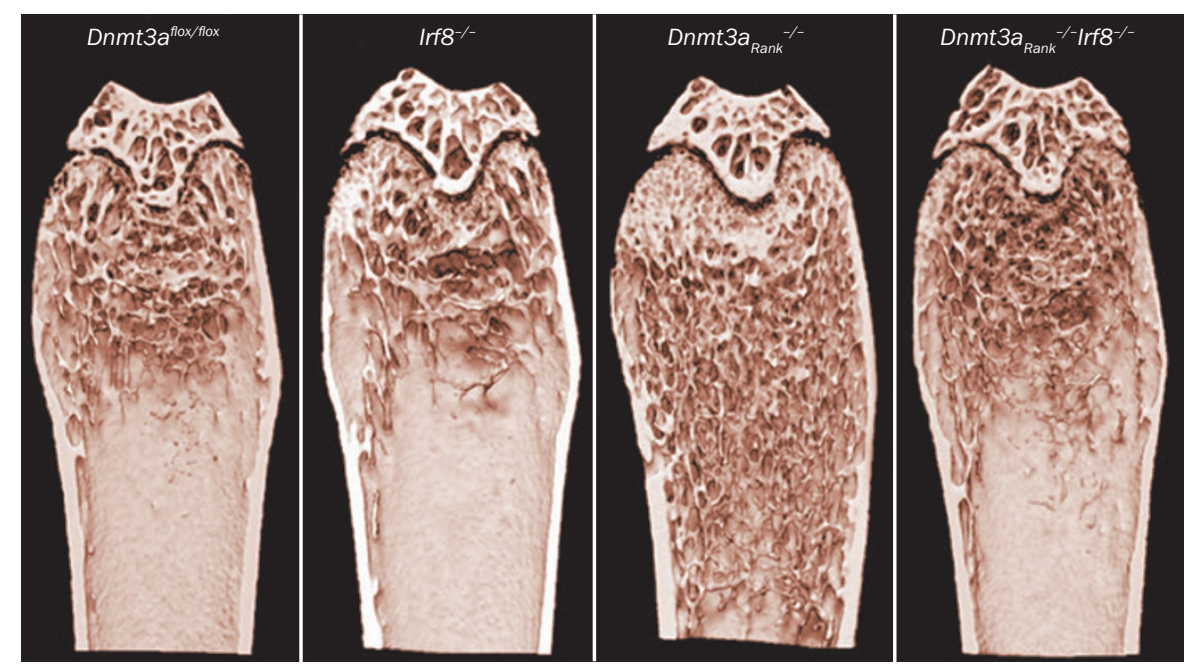

$\mu \mathrm{CT}$ images of the femurs of 10-week-old control, Irf8 ${ }^{-/-}$, Dnmt $3 a_{\text {Rank }^{-/-}}$and Dnmt3a $a_{\text {Rank }^{-1-I}}$ Iff $^{-/-}$male mice. Image from Nishikawa, K. et al. Nat. Med. doi:10.1038/nm.3774

The next step was to determine the therapeutic effect of the Dnmt3a inhibitor theaflavin-3,3'-digallate (TF-3) in an osteoporosis model. Although injection of TF-3 protected control mice against OVXinduced bone loss and increase in osteoclast number (in comparison with salineinjected OVX mice), “TF-3 had a negligible effect on bone mass and osteoclast number in Dnmt3a knockout OVX mice, indicating that TF-3 exerts its effect on Dnmt3a in osteoclasts," reports Nishikawa. "Our data suggest that inhibition of Dnmt3amediated epigenetic regulation is a potentially effective strategy for preventing bone loss."

A comprehensive search for genes regulated by Dnmt3a-mediated transcriptional repression during osteoclastogenesis led the researchers to focus on Irf8, encoding interferon regulatory factor 8 (Irf-8), which is known to block osteoclast differentiation. Experiments in bone-marrow derived monocyte-macrophage precursor cells (BMMs) demonstrated that Dnmt3amediated DNA methylation during osteoclastogenesis downregulates Irf8 expression.
The investigators then generated double-knockout Dnmt $3 a_{\text {Rank }}{ }^{-1-}$ Irf $8^{-/}$ mice, which they found to have low bone mass compared with Dnmt $3 a_{\text {Rank }}{ }^{-/-}$mice, in association with a high number of osteoclasts. In addition, in vitro osteoclast differentiation was accelerated in Dnmt3a $a^{-1-}$ Irf8 $8^{-/}$BMMs in comparison with $D n m t 3 a^{-/}$cells. These results suggest that Irf-8 acts downstream of Dnmt3a. Further experiments demonstrated that increased production of the methyl donor S-adenosylmethionine, coupled to a RANKL-induced shift toward oxidative metabolic processes in osteoclasts, has an important role in Irf8 regulation.

"The present results show that DNA methylation, which was originally targeted for cancer therapies that inhibit tumour growth, is also emerging as a promising therapeutic target for treating bone resorptive disorders such as osteoporosis," concludes Nishikawa.

\section{Sarah Onuora}

Original article Nishikawa, K. et al. DNA methyltransferas 3 a regulates osteoclast differentiation by coupling to an S-adenosylmethionine-producing metabolic pathway. Nat. Med. doi:10.1038/nm.3774 